\title{
A Pitfall in Some of the Robust Stability Literature
}

\author{
B. R. Barmish*, P. P. Khargonekar ${ }^{\dagger}$, Z. C. Shi ${ }^{*}$ and R. Tempo ${ }^{\ddagger}$ \\ * Department of Electrical and Computer Engineering \\ University of Wisconsin-Madison \\ Madison, Wisconsin 53706 \\ ${ }^{\dagger}$ Department of Electrical Engineering and Computer Science \\ University of Michigan \\ Ann Arbor, Michigan 48109 \\ ${ }^{\ddagger}$ CENS-CNR, Politecnico di Torino \\ Corso Duca degli Abruzzi 24 \\ 10129 Torino (Italy)
}

\begin{abstract}
For systems with structured real perturbations, it is shown that the robustness margin for stability can be a discontinuous function of the problem data. This raises the possibility that a certain type of "ill posedness" pervades much of the robust stability literature.
\end{abstract}

\section{Introduction}

Consider a linear control system with a transfer function or state space description parameterized in terms of a vector of uncertain parameters $q \in \mathbf{R}^{\ell}$. A fundamental problem addressed in literally hundreds of papers is: Determine the maximum uncertainty bound (call it $r_{m a x}^{*}$ ) such that the system is stable for all $q \in \mathbf{R}^{\ell}$ with $\|q\| \leq r_{\max }^{*}$. Note that the chosen norm for $q$ is almost always $\ell^{2}$ or $\ell^{\infty}$ and $r_{\max }^{*}$ is generally called the robustness margin; see Section 2 for a precise definition.

In many cases, a slightly different formulation of the problem above is considered; i.e., given an uncertainty bound $r>0$, determine if the systern is stable for all $q \in \mathbf{R}^{\ell}$ with $\|q\| \leq r$. In this case, only a "yes" or "no" answer is required. In the sequel, all analysis is carried out in the robustness margin framework but it should be noted that the consequences apply equally well to this alternative yes/no formulation; e.g., see the discussion associated with the example in Section 3.

The simple paradigms above apply to much of the robust stability in the literature - ranging from real $\mu$ as in Doyle (1985) to the post-Kharitonov literature (see Barmish (1988) and Jury (1988) for reviews of the continuous-time and discretetime cases respectively), to polytope stability problems as in Bartlett, Hollot and Huang (1988) and to the theory dealing with spherical uncertainty sets; e.g., see Biernacki, Hwang and Bhattacharryya (1987).

Our main objectives in this paper are twofold: The first objective is to show that the robustness margin $r_{\max }^{*}$ is not necessarily continuous with respect to the problem data; the notion of problem data will be more fully explained in the sequel. This discontinuity phenomenon is seen to be independent of the computational algorithm used to actually find $r_{m a x}^{*}$; i.e., we raise the possibility that the universally accepted definition of robustness margin is in a sense defective. Matters are further complicated by the fact that at the point of discountinuity, the robustness margin may be much smaller than at neighboring points. This may lead to potentially deceptive conclusions.

The second objective of this paper is to demonstrate that there may be severe consequences of the discontinuity phenomenon when numerical computation of $r_{\max }^{*}$ is attempted. To this end, we consider a certain frequency sweeping method as an example of a specific technique used for computation of the robustness margin; e.g., see Biernacki, Hwang and Bhattacharryya (1987), Saridereli and Kern (1987), de Gaston and Safonov (1988), Hinrichsen and Pritchard (1988), Vicino (1988) and Barmish (1989) as examples of papers which use a frequency sweep.

It is felt that for simple uncertainty structures, it may be possible to perform some sort of apriori check for discontinuity. For more complicated uncertainty structures, however, it is unclear if a discontinuity can be "flagged." The example given in Section 4 illustrates this point.

\section{Notation and Definition of the Robustness Margin}

We consider polynomials with real coefficients $a_{i}$ which depend continuously on a vector of uncertain parameters $q \in \mathbf{R}^{\ell}$ whose $i$-th component is $q_{i}$. To denote the dependence of $a_{i}$ on $q$, we write $a_{i}(q)$. Hence, we take an uncertain polynomial to be of the form

$$
p(s, q)=s^{n}+\sum_{i=0}^{n-1} a_{i}(q) s^{i}
$$

where the specific dependence of the $a_{i}(q)$ on $q$ will be stated in each example. In Section $3, a_{i}(q)$ is affine linear and in Section $4, a_{i}(q)$ is multilinear. When $q=0$, we obtain the so-called nominal polynomial $p(s, 0)$. In the subsequent discussions about robustness margins, without loss of generality, it is assumed that $p(s, 0)$ is strictly stable; i.e., its roots lie in the strict left half plane. This condition is satisfied in all the examples illustrating the discontinuity phenomenon. 
A bounding set for the vector of uncertain parameters $q$ will be a box or sphere parameterized by its radius $r$; this ball is denoted as $Q_{r}$. For the case of boxes, $Q_{r}$ is described by

$$
\left|q_{i}\right| \leq r ; i=1,2, \cdots, \ell
$$

and for the case of spheres, $Q_{r}$ is described by

$$
\left(\sum_{i=1}^{\ell} q_{i}^{2}\right)^{\frac{1}{2}} \leq r .
$$

Robustness Margin: In accordance with the discussion in Section 1, the robustness margin (for stability) is given by

$r_{\text {max }}^{*} \doteq \sup \left\{r: p(s, q)\right.$ is strictly stable for all $\left.q \in Q_{r}\right\}$.

Note that the discussion to follow can easily be modified so as to apply to other notions of strict stability. For example, strict stability can be defined in terms of the open unit disk.

Dependence on Problem Data: In each of the examples to follow, the integer $\ell=\operatorname{dim} q$ and $n=\operatorname{deg} p(s, q)$ are held fixed and problem data consists of the coefficient functions $a_{0}(\cdot)$ $a_{1}(\cdot), \ldots, a_{n-1}(\cdot)$. To illustrate the discontinuity phenomenon, we use a finite-dimensional space for this problem data. That is, each $a_{i}(\cdot)$ is viewed as a mapping on data vectors $d \in \mathbf{R}^{p}$ to continuous functions of $q$. To illustrate, a family of problems might be described by $p=6$ and

$$
p(s, q)=s^{2}+\left(d_{1}+d_{2} q_{1}+d_{3} q_{2}\right) s+\left(d_{4}+d_{5} q_{1}+d_{6} q_{2}\right) .
$$

A specific robustness margin problem is obtained with $d_{1}=2, d_{2}=1, d_{3}=4, d_{4}=3, d_{5}=6$ and $d_{6}=12$. This leads to

$$
p(s, q)=s^{2}+\left(2+q_{1}+4 q_{2}\right) s+\left(3+6 q_{1}+12 q_{2}\right) .
$$

Within this framework, two problems are "close together" if their associated data vectors (call them $d^{1}$ and $d^{2}$ ) are close together in some suitably defined norm on $\mathbf{R}^{p}$; i.e., $\left\|d^{1}-d^{2}\right\|$ is small.

To denote dependence on $d$, we write $p_{d}(s, q)$ and $r_{\max }^{*}(d)$ in lieu of $p(s, q)$ and $r_{\max }^{*}$ respectively. We are now prepared to expose the discontinuity phenomenon.

\section{Example of Discontinuity for Affine Linear Perturbation Structure}

Consider

$$
\begin{aligned}
p_{d}(s, q)= & s^{4}+\left(d_{1}+d_{2} q_{2}\right) s^{3}+\left(d_{3}+d_{4} q_{1}\right) s^{2} \\
& +\left(d_{5}+d_{6} q_{2}\right) s+\left(d_{7}+d_{8} q_{1}+d_{9} q_{2}\right) .
\end{aligned}
$$

For the case when $Q_{r}$ is a sphere, we claim that there is a sequence of $d$ vectors, call it $\langle d(n)\rangle_{n=1}^{\infty}$, which converges to the data point $d^{*}$ with components $d_{1}^{*}=4, d_{2}^{*}=-1, d_{3}^{*}=8$, $d_{4}^{*}=-2, d_{5}^{*}=12, d_{6}^{*}=-3, d_{7}^{*}=9, d_{8}^{*}=-1$ and $d_{9}^{*}=-5$, and moreover,

$$
1.77 \approx \lim _{n \rightarrow \infty} r_{\max }^{*}(d(n))>r_{\max }^{*}\left(d^{*}\right) \approx 0.85 .
$$

That is, the robustness margin is discontinuous at the data point $d^{*}$. This implies that for infinitesimally small deviations away from $d^{*}$, the robustness margin is $r_{\text {max }}^{*}(d(n)) \approx 1.77$, but precisely at $d^{*}$, the robustness margin becomes $r_{\max }^{*}\left(d^{*}\right) \approx 0.85$ which is much smaller. This example illustrates the "false sense of security," one may obtain using $r_{m a x}^{*}$. To further dramatize this point, Figure 1 provides a plot of one of the "rightmost" root of $p_{d(n)}\left(s, q^{*}\right)$ with $q_{1}^{*}=\frac{3}{5}$ and $q_{2}^{*}=-\frac{3}{5}$; of course, the other rightmost root is the complex conjugate.

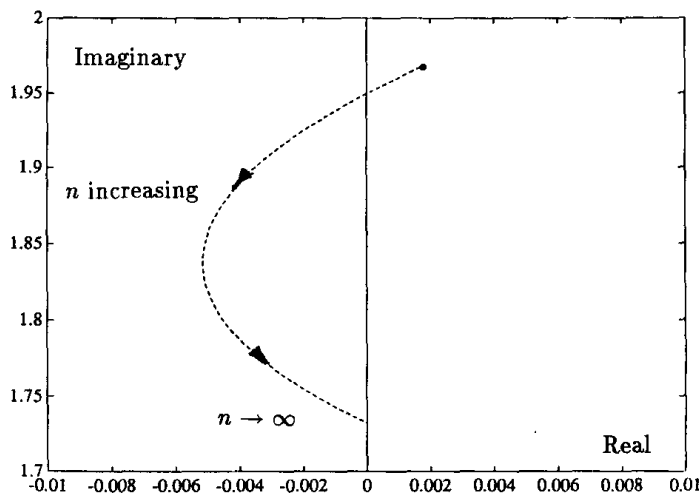

Figure 1: One of Rightmost Roots of $p_{d(n)}\left(s, q^{*}\right)$

Notice that as $n$ becomes large, $r_{\max }^{*}(d(n))$ tends to 1.77 and the root is approaching the imaginary axis. However, the $q^{*}$ vector being used has norm 0.85 . That is, $p_{d(n)}\left(s, q^{*}\right)$ is "nearly" destabilized by a $q$ vector whose norm is 0.85 despite the fact that the predicted margin is 1.77 . We will also demonstrate the same type of discontinity phenomenon when $Q_{r}$ is a box.

We will establish (1) using the so-called "frequency sweep" method to obtain a closed form for $r_{m a x}^{*}$. Indeed, the frequency sweep method is based on the following idea: At each frequency $\omega \in \mathbf{R}$, we compute a quantity $r_{\max }(d, \omega)$ representing the maximal value of the uncertainty bound $r$ such that $p_{d}(s, q)$

has no roots at $s=j \omega$. That is, let

$$
r_{\max }(d, \omega) \doteq \sup \left\{r: p_{d}(j \omega, q) \neq 0 \text { for all } q \in Q_{r}\right\} .
$$

To obtain the robustness margin, we take the infimum of $r_{\max }(d, \omega)$ over all frequencies; i.e., for fixed $d$,

$$
r_{\max }^{*}(d) \doteq \inf _{\omega} r_{\max }(d, \omega) .
$$

Now for $d=d^{*}$, we compute $r_{\text {max }}^{*}\left(d^{*}\right)$ using both boxes and spheres for $Q_{r}$. Subsequently, the result will be interpreted in terms of the cited literature. Since there are only two perturbations $q_{1}$ and $q_{2}, r_{m a x}\left(d^{*}, \omega\right)$ can be obtained in closed form. This is accomplished by setting

$$
\operatorname{Re} p_{d^{\bullet}}(j \omega, q)=\omega^{4}-\left(8-2 q_{1}\right) \omega^{2}+\left(9-q_{1}-5 q_{2}\right)
$$

and

$$
\text { Im } p_{d^{*}}(j \omega, q)=-\left(4-q_{2}\right) \omega^{3}+\left(12-3 q_{2}\right) \omega
$$

to zero and solving for $q_{1}$ and $q_{2}$ as a function of $\omega$. At frequencies where the two equations are linearly dependent, one seeks the smallest norm solution - when $Q_{r}$ is a sphere, the euclidean norm is used and when $Q_{r}$ is a box the max norm is used. For these two cases, we summarize the results of this lengthy but straightforward hand calculation below. 
Case 1: $Q_{r}$ is a sphere in $\mathbf{R}^{2}$.

$$
r_{\max }\left(d^{*}, \omega\right)= \begin{cases}\frac{9 \sqrt{26}}{26} & \text { if } \omega=0 \\ +\infty & \text { if } \omega=\frac{\sqrt{2}}{2} \\ \frac{3 \sqrt{2}}{5} & \text { if } \omega=\sqrt{3} \\ R\left(d^{*}, \omega\right) & \text { otherwise }\end{cases}
$$

where

$$
R\left(d^{*}, \omega\right)=\sqrt{\left(\frac{\omega^{4}-8 \omega^{2}-11}{2 \omega^{2}-1}\right)^{2}+16} .
$$

A plot of margin $r_{\max }\left(d^{*}, \omega\right)$ versus $\omega$ is depicted in Figure 2 .

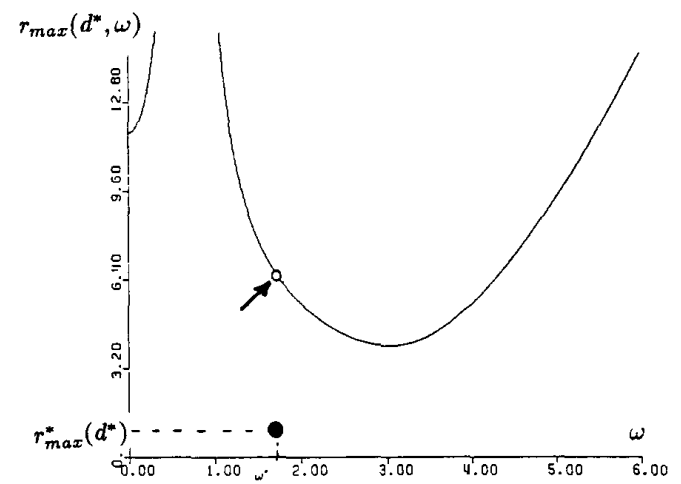

Figure 2: A Plot of $r_{\max }\left(d^{*}, \omega\right)$ Versus $\omega$

The global minimum of $r_{\max }\left(d^{*}, \omega\right)$ occurs at $\omega^{*}=\sqrt{3}$ which is an isolated point of discontinuity. In this case, from (2), we obtain the robustness margin

$$
r_{m a x}^{*}\left(d^{*}\right)=r_{m a x}\left(d^{*}, \sqrt{3}\right)=\frac{3 \sqrt{2}}{5} \approx 0.85 .
$$

Finally, to arrive at inequality (1), we consider the sequence $\langle d(n)\rangle_{n=1}^{\infty}$ converging to $d^{*}$ defined by $d_{i}(n)=d_{i}^{*}$ for $i \neq 5$ and $d_{5}(n)=12+\frac{1}{n}$. Hence, we have

$$
\begin{aligned}
p_{d(n)}(s, q)= & s^{4}+\left(4-q_{2}\right) s^{3}+\left(8-2 q_{1}\right) s^{2} \\
& +\left(12+\frac{1}{n}-3 q_{2}\right) s+\left(9-q_{1}-5 q_{2}\right)
\end{aligned}
$$

and it is straightforward to verify that for each $n, r_{\max }(d(n), \omega)$ is no longer discontinuous for $\omega>0$. In fact, as $n$ increases, we now have $r_{\max }^{*}(d(n)) \rightarrow r_{\max }(d(n), 0) \rightarrow 1.77$. Hence, it follows that

$$
\lim _{n \rightarrow \infty} r_{\max }^{*}(d(n)) \approx 1.77
$$

Recalling, however, that

$$
r_{\max }^{*}\left(d^{*}\right) \approx 0.85
$$

we conclude that

$$
\lim _{n \rightarrow \infty} r_{\max }^{*}(d(n))>r_{\max }^{*}\left(d^{*}\right) .
$$

Further Discussion of Frequency Sweep Method: Note that exact arithmetic was used above. When performing digital computations however, a serious problem can arise: Even an infinitely precise digital computation over any finite set of frequencies may lead to an erroneous result. To see this, let $d=d^{*}$ and notice that as $\omega \rightarrow \sqrt{3}$, the left and right hand limits are given by

$$
r_{\max }\left(d^{*}, \sqrt{3}^{-}\right)=r_{\max }\left(d^{*}, \sqrt{3}^{+}\right) \approx 6.56 .
$$

Hence, if an infinite precision computation excludes the critical point $\omega=\sqrt{3}$, one obtains the "phony" local minimum at $\omega \approx 3.0$. That is, from (2), it follows that

$$
r_{\max }\left(d^{*}, 3\right) \approx 4.0 \text {. }
$$

The frequency sweep method also dictates that we must examine $\omega=0$ separately. Comparing the $\omega=0$ analysis with the phony minimum obtained for $\omega>0$, we end up with the erroneous result

$$
\inf _{\omega \neq \sqrt{3}} r_{\max }\left(d^{*}, \omega\right)=r_{\max }\left(d^{*}, 0\right)=\frac{9 \sqrt{26}}{26} \approx 1.77 .
$$

Since the discontinuity at $\omega=\sqrt{3}$ is isolated, it is generally unclear to someone performing computations that this pathology is occuring. That is, in the neighborhood of the critical frequency, $r_{\max }\left(d^{*}, \omega\right)$ may appear to be well behaved and a "warning" may not be obtained when performing computation. This may lead to a final computed value of $r_{\max }^{*}\left(d^{*}\right)$ which is seriously in error.

We state emphatically that the issue being raised here is not one of numerical roundoff. Even exact arithmetical calculations at an arbitrarily large but finite number of frequencies will not alleviate this difficulty. Our conclusion is that there is a fundamental issue at the basic level of the theory.

Examination of the frequency sweeping literature reveals that authors are fully aware that at $\omega=0$, discontinuities in $r_{\max }(d, \omega)$ are an issue - this discontinuity is easily handled by a separate calculation of $r_{\max }(d, 0)$. There is also an awareness that $r_{\max }(d, \omega)$ can blow up to infinity. Once again, their analyses remain intact if $r_{\max }(d, \omega) \rightarrow \infty$ because we are concerned with the minimum value of $r_{\max }(d, \omega)$. However, with regard to isolated discontinuities which may occur at "weird" frequencies, only a few sentences in de Gaston and Safonov (1988) and Fu (1988) raise the possibility that these authors may have been aware of this problem. At any rate, their expositions are couched in terms of "fixes" for numerical computation rather than the need for modification of the underlying definition of robustness margin. In this regard, recall that the discontinuity phenomenon is independent of computing method. Problems which arise using the frequency sweeping method should rightfully be viewed as one particular manifestation of a more serious problem.

Case 2: $Q_{r}$ is a box in $\mathbf{R}^{2}$.

$$
r_{\max }\left(d^{*}, \omega\right)= \begin{cases}\frac{3}{2} & \text { if } \omega=0 \\ +\infty & \text { if } \omega=\frac{\sqrt{2}}{2} \\ \frac{3}{5} & \text { if } \omega=\sqrt{3} \\ R\left(d^{*}, \omega\right) & \text { otherwise }\end{cases}
$$

where

$$
R\left(d^{*}, \omega\right)=\max \left\{\left|\frac{\omega^{4}-8 \omega^{2}-11}{2 \omega^{2}-1}\right|, 4\right\} .
$$

The same phenomenon occurs as in Case 1. Namely, the robustness margin is obtained as

$$
r_{\max }^{*}\left(d^{*}\right)=r_{\max }\left(d^{*}, \sqrt{3}\right)=\frac{3}{5}
$$

but there is a data sequence $\langle d(n)\rangle_{n=1}^{\infty}$ which converges to $d^{*}$ such that

$$
\lim _{n \rightarrow \infty} r_{m a x}^{*}(d(n))=\frac{3}{2}
$$


When numerical computations are performed, the same problems arise as in the spherical case. Namely, the left and right hand limits are given by

$$
r_{\max }\left(d^{*}, \sqrt{3}^{-}\right)=r_{\max }\left(d^{*}, \sqrt{3}^{+}\right)=5.20 .
$$

Hence, if a frequency grid excludes the critical point $\omega^{*}=\sqrt{3}$, one obtains the erroneous result

$$
r_{\max }^{*}\left(d^{*}\right)=r_{\max }\left(d^{*}, 0\right)=\frac{3}{2}
$$

Remarks: In order to provide an interface with papers which deal with control systems rather than polynomials, consider the plant

$$
G_{d^{*}}(s, q)=4 \frac{\left(1-q_{2}\right) s^{3}+3 s^{2}+\left(3-q_{2}\right) s+\left(2-q_{2}\right)}{s^{4}+3 q_{2} s^{3}-\left(4+2 q_{1}\right) s^{2}+q_{2} s+\left(1-q_{1}-q_{2}\right)}
$$

with unity feedback. The closed loop polynomial for this system is readily verified to be the same polynomial $p_{d^{*}}(s, q)$ which we just considered above. The results of this section can also be interpreted in terms of the yes/no problem formulation discussed in the introduction. To illustrate, consider Case 1 above with given uncertainty bound $r \in(0.85,1.77)$. Now, the following phenomenon arises. When $d=d^{*}$, the answer to the robust stability question is "no" but infinitesimally close to $d^{*}$, the answer is "yes."

\section{Example of Discontinuity for Multilinear Perturbation Structure}

For the so-called multilinear perturbation structure, we now exhibit the same discontinuity phenomenon. Indeed, we take

$p_{d}(s, q)=s^{3}+d_{1} s^{2}+\left(d_{2}+d_{3} q_{1}\right) s+\left(d_{4} q_{1} q_{2}+d_{5} q_{1}+d_{6} q_{2}+d_{7}\right)$

with discontinuity point $d^{*}$ described by $d_{1}^{*}=3, d_{2}^{*}=3, d_{3}^{*}=1$, $d_{4}^{*}=1, d_{5}^{*}=-1, d_{6}^{*}=1$ and $d_{7}^{*}=5$. This leads to

$$
p_{d^{*}}(s, q)=s^{3}+3 s^{2}+\left(3+q_{1}\right) s+\left(q_{1} q_{2}-q_{1}+q_{2}+5\right) .
$$

As in the previous section, we compute $r_{\max }\left(d^{*}, \omega\right)$ in closed form. By setting real and imaginary parts of $p_{d^{*}}(j \omega, q)$ to zero, we obtain $r_{\max }\left(d^{*}, \omega\right)$ in the same manner as described in Section 3.

Case 1: $Q_{r}$ is a sphere in $\mathbf{R}^{2}$

$$
r_{\max }\left(d^{*}, \omega\right)= \begin{cases}\approx 2.05 & \text { if } \omega=0 \\ 1 & \text { if } \omega=\sqrt{2} \\ R\left(d^{*}, \omega\right) & \text { otherwise }\end{cases}
$$

where

$$
R\left(d^{*}, \omega\right)=\sqrt{\omega^{4}-6 \omega^{2}+25} .
$$

The troublesome discontinuity occurs at the critical frequency $\omega^{*}=\sqrt{2}$. This discontinuity is not only isolated, but it is also at the global minimum of $r_{\max }\left(d^{*}, \omega\right)$. Now, from (3), we obtain the robustness margin

$$
r_{\max }^{*}\left(d^{*}\right)=r_{\max }\left(d^{*}, \sqrt{2}\right)=1 .
$$

However, as in the previous example, evaluation of $r_{\max }(d, \omega)$ over any set of frequencies which excludes $\omega^{*}=\sqrt{2}$ leads to the erroneous result
Analogous to the previous section, note that one can easily construct a data sequence $\langle d(n)\rangle_{n=1}^{\infty}$ which converges to $d^{*}$ and has the property that

$$
\lim _{n \rightarrow \infty} r_{\max }^{*}(d(n)) \approx 2.05 .
$$

That is,

$$
\lim _{n \rightarrow \infty} r_{\max }^{*}(d(n))>r_{\max }^{*}\left(d^{*}\right) .
$$

Case 2: $Q_{r}$ is a box in $\mathbf{R}^{2}$.

$$
r_{\max }\left(d^{*}, \omega\right)= \begin{cases}\approx 1.45 & \text { if } \omega=0 \\ 1 & \text { if } \omega=\sqrt{2} \\ R\left(d^{*}, \omega\right) & \text { otherwise }\end{cases}
$$

where

$$
R\left(d^{*}, \omega\right)=\max \left\{\left|\omega^{2}-3\right|, 4\right\} .
$$

Now, from (4) we obtain the "true" robustness margin

$$
r_{\max }^{*}\left(d^{*}\right)=r_{\max }\left(d^{*}, \sqrt{2}\right)=1 .
$$

As in Case 1, evaluation of $r_{\max }^{*}\left(d^{*}, \omega\right)$ over any set of frequencies which excludes $\omega^{*}=\sqrt{2}$ leads to the erroneous result

$$
r_{\text {max }}^{*}\left(d^{*}\right) \approx 1.45 \text {. }
$$

Again, using an appropriate data sequence $\langle d(n)\rangle_{n=1}^{\infty}$, it is easy to demonstrate that $r_{m a x}^{*}(d)$ is discontinuous at $d=d^{*}$.

\section{Conclusion}

Although the discontinuity examples given here were for continuous-time, it is also quite easy to illustrate the same pathology for the discrete-time case. It is important to note, however, that not all "uncertainty structures" exhibit the discontinuity phenomenon. For example, suppose that $Q_{r}$ is a box or a sphere and $p_{d}(s, q)$ has a so-called independent perturbation structure; e.g., see Kharitonov (1978) or Soh, Berger and Dabke (1985). That is, each component $q_{i}$ of $q$ enters linearly into only one coefficient of $p_{d}(s, q)$. For these cases, it can be shown that $r_{\max }^{*}(d)$ is continuous with respect to $d$. Furthermore, for fixed $d$, the frequency sweep method results in a function $r_{\max }(d, \omega)$ which has no points of discontinuity other than $\omega=0$.

\section{Acknowledgements}

This work was performed while B. R. Barmish was a Senior NATO Guest Fellow at CENS-CNR, Torino (Italy). The support of Professor A. R. Meo is gratefully acknowledged. Partial support of this project was also provided by the U.S. National Science Foundation under Grants ECS-8612948 and ECS-8451519, by the U.S. Air Force Office of Scientific Research under Grant AFOSR-88-0020 and by Honeywell, Boeing and General Electric. 


\section{References}

Barmish, B. R. (1988). New Tools for Robustness Analysis, Proceedings of the IEEE Conference on Decision and Control, Austin.

Barmish, B. R. (1989). A Generalization of Kharitonov's Four Polynomial Concept for Robust Stability Problems with Linearly Dependent Coefficient Perturbations, IEEE Transactions on Automatic Control, vol. AC-34, no. 2, pp. 157-165.

Bartlett, A. C., C. V. Hollot and L. Huang (1988). Root Locations of an Entire Polytope of Polynomials: It Suffices to Check the Edges, Mathematics of Control, Signals and Systems, vol. 1, pp. 61-71.

Biernacki, R. M., H. Hwang and S. P. Bhattacharryya (1987). Robust Stability with Structured Real Parameter Perturbations, IEEE Transactions on Automatic Control, AC-32, pp. 495506.

de Gaston, R. R. E. and M. G. Safonov (1988). Exact Calculation of the Multiloop Stability Margin, IEEE Transactions on Automatic Control, AC-33, pp. 156-171.

Doyle, J. C. (1985). Structured Uncertainty in Control System Design, Proceedings of the IEEE Conference on Decision and Control, Ft. Lauderdale.

Fu, M. (1988). New Criteria and Algorithms for Robust Stability of Families of Polynomials, Robustness in Identification and Control, (M. Milanese, R. Tempo and A. Vicino Eds.), Plenum Press.

Jury, E. I. (1988). Robustness of Discrete Systems: A Review, to appear.

Hinrichsen, D. and A. S. Pritchard (1988). New Robustness Results for Linear Systems under Real Perturbations, Proceedings of the IEEE Conference on Decision and Control, Austin. Kharitonov, V. L. (1978). Asymptotic Stability of an Equilibrium Position of a Family of Systems of Linear Differential Equations, Differential'nye Uraveniya, vol. 14, no. 11, pp. 14831485.

Saridereli, M. K. and F. J. Kern (1987). The Stability of Polynomials under Correlated Coefficient Perturbations, Proceedings of the IEEE Conference on Decision and Control, Los Angeles.

Soh, C. B., C. S. Berger and K. P. Dabke (1985). On the Stability Property of Polynomials with Perturbed Coefficients, IEEE Transactions on Automatic Control, AC-30, pp. 10331036.

Vicino, A. (1988). Some Results on Robust Stability of Discrete Time Systems, IEEE Transactions on Automatic Control, AC-33, pp. 844-847. 